\title{
NEW PRAESTOCHRYSIS AND NOTES ON DESCRIBED SPECIES FROM THE ORIENTAL REGION (HYMENOPTERA, CHRYSIDIDAE)*
}

\author{
By R. M. Bohart, Department of Entomology \\ University of California, Davis, CA 95616
}

The genus Praestochrysis Linsenmaier is distinguished by having 5 distinct teeth on tergum III and a rather stout antenna. In any case the latter has flagellomere I less than 3 times as long as broad. Bohart (1986, Insecta Mundi 1:148-154) gave a key to species of the Ethiopian Region. One additional species, lamborni, is here described from that region. It keys out to couplet 6 in the 1986 paper but differs in having no transverse frontal carina.

Abbreviations used in the following descriptions are: F-I, etc., flagellomeres; TFC, transverse frontal carina; MOD, median ocellus diameter; T-I, etc., terga; S-II, sternum.

\section{Praestochrysis lamborni Bohart, new species}

Male holotype: Length $7.5 \mathrm{~mm}$. Body moderately stout, green, midscutum mostly purple, F-I-II green in front, wings weakly stained. Punctures moderately small and close. Head $1.25 \times$ as broad as long (fig. 2a); F-I 1.7X as long as broad (fig. 2d), F-II as broad as long, F-V twice as broad as long; scapal basin short and broad, polished above and in middle one-third below, rest punctate, frons $1.8 \times$ as broad at malar space as at its least width; TFC absent, midocellus lidded, malar and subantennal spaces each about 1 MOD; pronotum about as long as scutellum, median groove weak; metanotum rounded (fig. 2c); mesopleuron edentate, scrobal and episternal sulci distinct; propodeal projection sharp, incurved behind; T-II without midcarina; T-III evenly convex before weakly developed pit row, lateral margin a little convex, 5 short, sharp distal teeth (fig. 2b); S-II spots small, triangular, nearly touching (fig. 2e).

Female. About as in male. T-III lateral margin slightly angled out, submedian teeth twice as long as others (fig. 2b).

*Manuscript received by the editor March 3, 1987. 
Holotype male, Malawi: Mangoche (Fort Johnston), I-14-34, emerged from flat moth cocoon on tree bark (W. A. Lamborn, OXFORD). Paratypes, 11 males, 29 females, same data as holotype (Oxford Museum, Bohart Museum); 3 males, 4 females, Malawi: Mzeze, $45 \mathrm{mi} \mathrm{n}$. Mangoche, emerged from flat moth cocoons on tree bark (W. A. Lamborn, Oxford Museum, Bohart Museum, other major museums).

Discussion. The rounded metanotum, 5 distal teeth on T-III, and broadened flagellum are characters found also in micromorpha Mocsáry and africanum Buysson. However, both of these species have a definite TFC. In addition the micromorpha F-I is only $1.4 \times$ as long as broad instead of 1.7 as in lamborni. Also, in africanum the malar space is more than 2 MOD instead of only 1 MOD, and the lateral margin of T-III is not at all bent outward. The species is named for W. A. Lamborn, who collected the long type series.

\section{Praestochrysis luzonae Bohart, new species}

Male holotype: Length $7.5 \mathrm{~mm}$. Body stout, green; deep purple across vertex, on scutum, and at base of T-III; T-III mostly bluish; F-I green in front; wings brown. Punctures coarse, close on vertex, widely separated by 4 smooth swellings on pronotum (fig. 3f), mostly a puncture diameter apart on rest of notum and terga. Head $1.55 \times$ as broad as long (fig. 3a), with prominent rugosities on vertex near eyes; F-I about twice as long as broad (fig. 3d), F-V 1.4X as broad as long; scapal basin short and broad, rather closely punctate; TFC broadly M-like (fig. 3a), branching above to nearly enclose midocellus and form a lid behind it; malar space 2.1 MOD; subantennal space $1 \mathrm{MOD}$; pronotum as long as scutellum, no median groove; metanotum with a flat, subtriangular mucro (fig. 3c); mesopleuron edentate but with prominent carinae below large scrobal sulcus, episternal sulcus deep and punctate; propodeal projection large, acute, slightly convex behind; T-II without midcarina; T-III evenly convex before weakly developed pit row (fig. 3b), pits unevenly shaped, mostly broader than long, lateral margin of III nearly straight, 5 sharp distal teeth, outer pair shortest; S-II spots rounded, touching (fig. 3e).

Female. Unknown.

Holotype male, Philippines: Luzon, Los Banos, IV-19-60 (A. Ojamin; U.S. National Museum, Washington). 

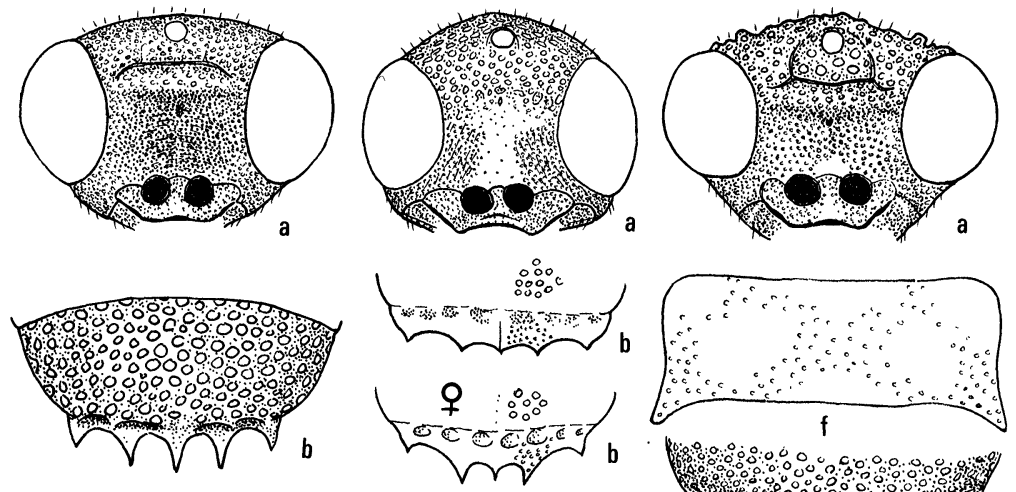

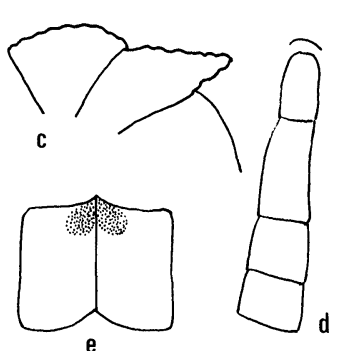

1. spinula

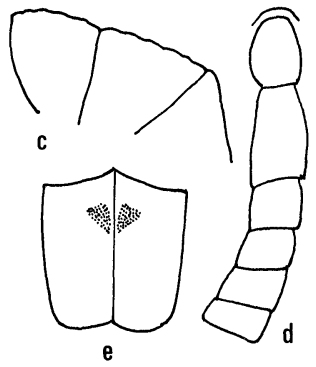

2. lamborni

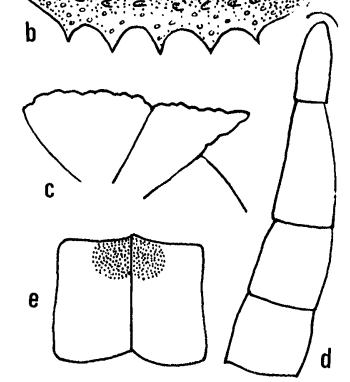

3. luzonae

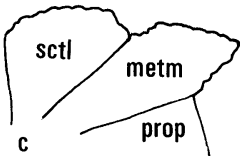

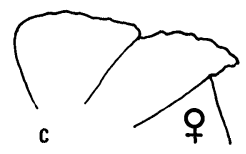

5. ribbei

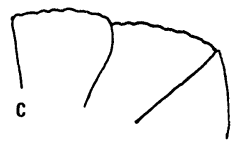

6. lusca

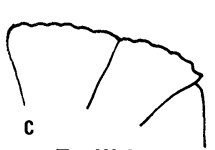

7. libita

Figures 1-7. Praestochrysis.

In all figs.: $a$, face; $b$, T-III dorsal; c, outline of scutellum, metanotum and propodeum, lateral; d, petiole and basal flagellomeres; e, sternum II; f, pronotum, dorsal; sctl, scutellum; metm, metanotum; prop, propodeum. Figures not drawn to scale, $2 \mathrm{~b}$ and $5 c$ are based on females, other figures based on males, those of 1-3 are from holotypes. 
Discussion. The 4 large and polished pronotal convexities are distinctive. Also, the rough projections behind the eyes have not been seen in any other Praestochrysis.

\section{Praetochrysis spinula Bohart, new species.}

Holotype male. Length $7 \mathrm{~mm}$. Body stout, blue green with extensive dark purple on vertex, notum, and terga, F-I green in front, wings light brown. Punctures medium coarse and fairly close on vertex and notum, coarse and separated by about a puncture diameter on terga. Head $1.5 \times$ as broad as long (fig. 1a); F-I 2.1 $\times$ as long as broad (fig. 1d), F-V 1.4X as broad as long; scapal basin rather finely punctate, punctures arranged in a somewhat crossridged pattern; TFC prominent, nearly straight, downturned laterally (fig. 1a), backward branches weak, midocellus not lidded; malar space 2.5 MOD; subantennal space $0.8 \mathrm{MOD}$; pronotum as long as scutellum, no median groove; metanotum with a subtriangular, distally rounded, dorsally flattened mucro (fig. 1c); mesopleuron with a well-formed scrobal sulcus, areolate below, episternal sulcus discernible but weak; propodeal projection stout, sharply pointed, evenly convex behind; T-II without a midcarina; T-III rather evenly and strongly convex, pit row obsolete, lateral margin of III a little convex, 5 sharp and slender teeth, outer pair shortest (fig. 1b); S-II spots rounded, touching (fig. 1e).

Female. Unknown.

Holotype male, Sri Lanka (DAVIS).

Discussion. The long T-III teeth, obsolete pit row, medially straight TFC, and flattened mucro distinguish this species. The Taiwanese basilacuna Sugihara has similar T-III teeth but the pit row is better developed, TFC is medially incised, and the metanotal mucro is longitudinally furrowed. Also, it is a larger species, 9-13.5 mm long.

\section{Notes on Oriental Praestochrysis}

While studying Oriental Praestochrysis, I have noted several characters of key value. The presence or absence of a metanotal projection is a prime distinguishing feature. A large such projection is often present in Praestochrysis (figs. 1, 3, 4, 5) as in basilacuna 
(Sugihara), crassiscuta (Mocsáry), fumipennis (Smith), lachesis (Mocsáry), luzonae Bohart, palawanensis (Mocsáry), ribbei (Mocsáry), sarawakensis (Mocsáry), shanghaiensis (Smith), spectabilis (Mocsáry), and spinula Bohart. On the other hand, several species have little or no metanotal projection (figs. 6, 7). These are amoenula (Mocsáry), furcifera (Bingham), libita (Buysson), and lusca (Fabricius). Other characters are the presence of 2 large mesopleural teeth (crassiscuta and spectabilis), a strong sublateral pronotal carina (lusca), pronotum with 4 smooth humps (luzonae), F-1 hardly longer than the pedicel (lachesis), and T-III teeth unusually long (basilacuna, spinula).

SUMMARY

Three new species of the chrysidid genus, Praestochrysis, are described and figured: lamborni from Malawi, luzonae from the Philippines, and spinula from Sri Lanka. Notes are given on previously named species from the Oriental Region. 

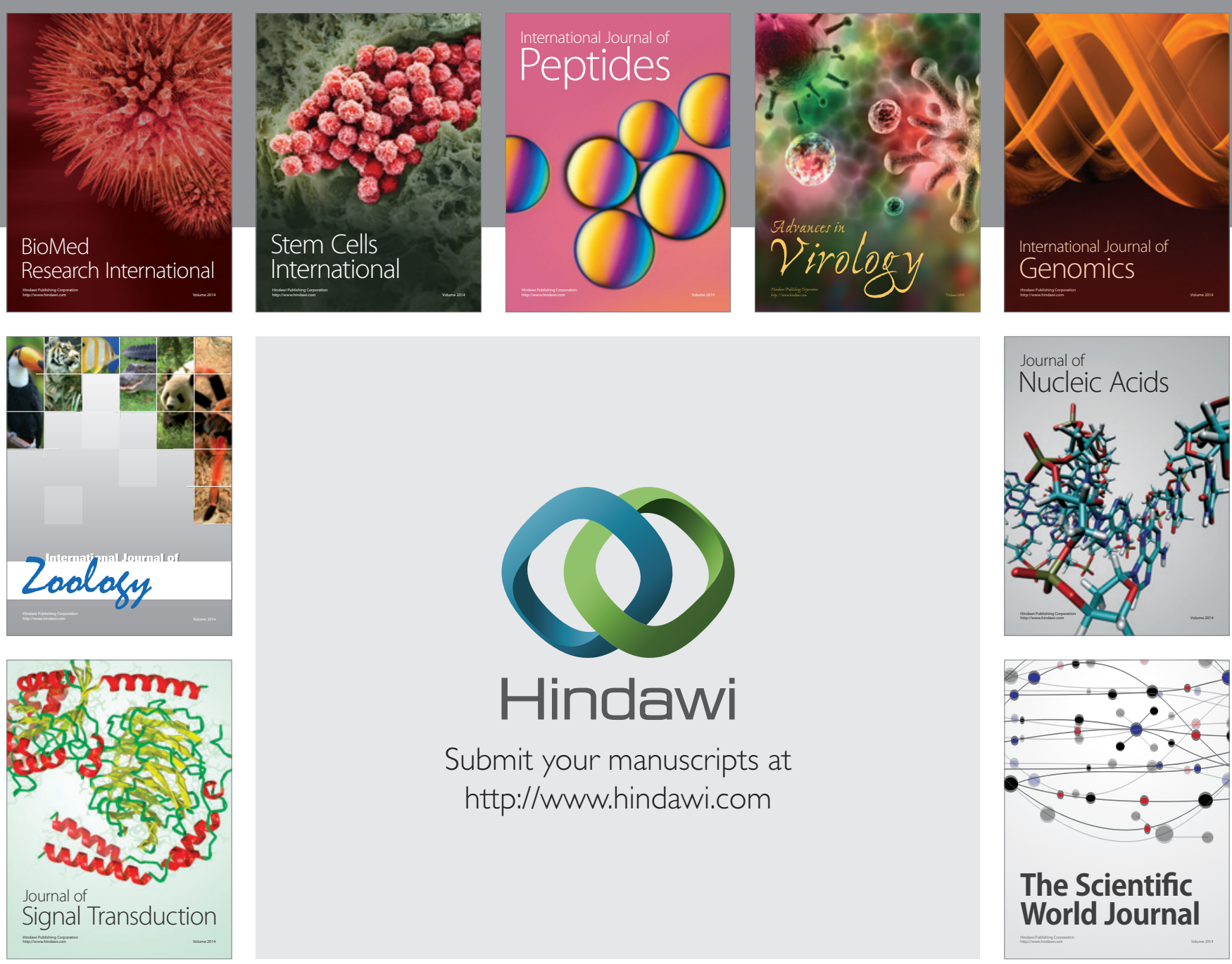

Submit your manuscripts at

http://www.hindawi.com
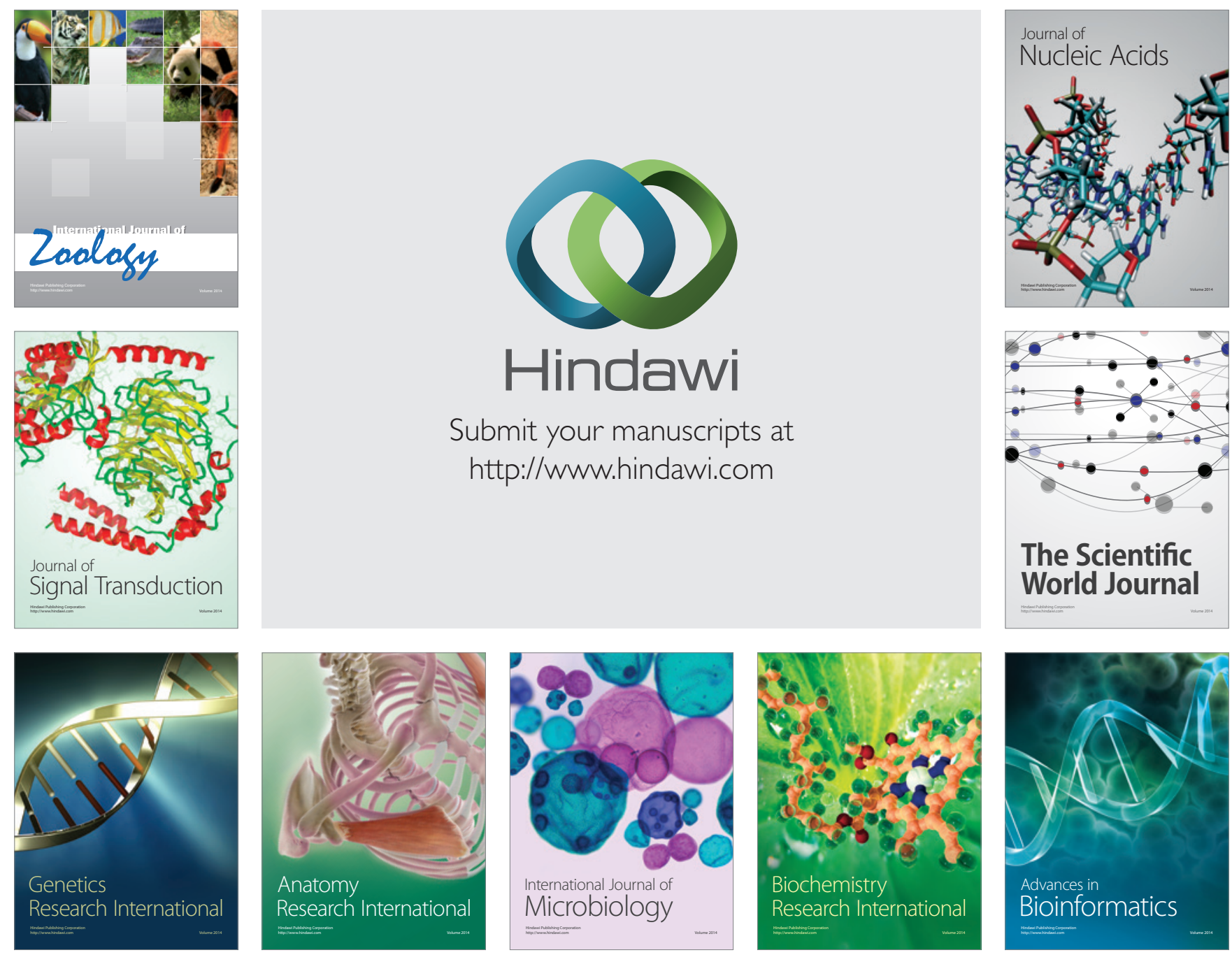

The Scientific World Journal
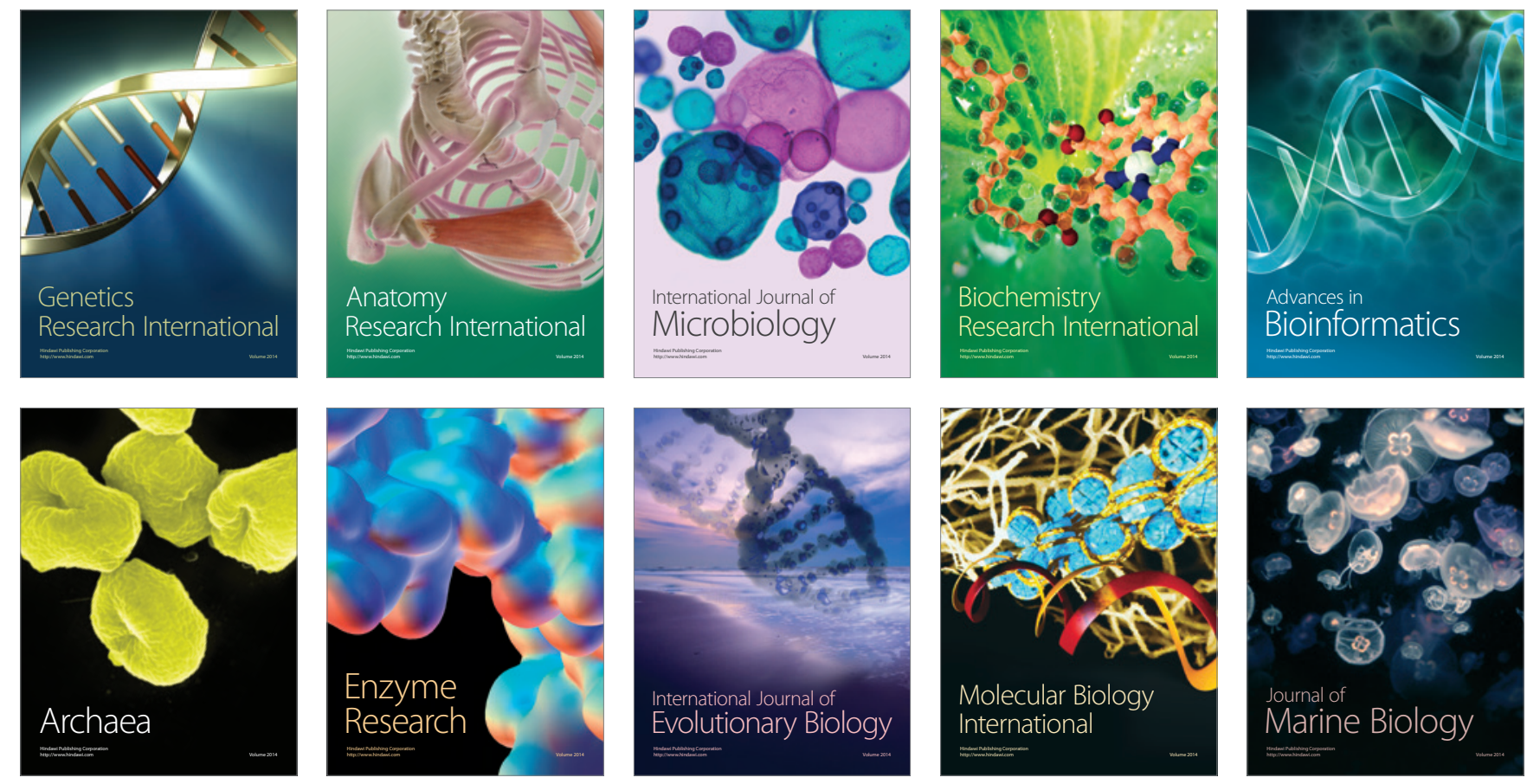